\title{
The dial-a-ride problem in the case of a patient transportation system in Brazil
}

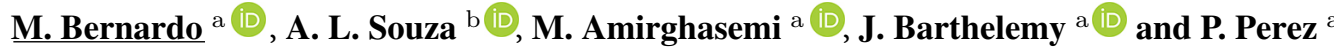 \\ aSMART Infrastructure Facility, University of Wollongong, Northfields Avenue, 2522 Wollongong, Australia \\ ${ }^{\mathrm{b}}$ Departamento de Computação, Universidade Federal de Ouro Preto, 35400000 Ouro Preto, Brazil
}

Email: marcella@uow.edu.au

\begin{abstract}
Despite its importance, the role played by logistics is often overlooked in health care. Patients often need to be transported to medical facilities and treatment units. In Brazil, the transportation department of municipal health care systems need to provide transport to patients from small municipalities to public hospitals located in large cities, as some medical services are only provided in these hospitals. The problem of transporting patients can be modelled as a Dial-a-Ride Problem (DARP). The DARP is a variant of the Vehicle Routing Problem (VRP) where a transportation request includes both a pick-up and drop-off locations and not only time window constraints but also other users' constraints are considered. The general goal of DARP is to calculate a route plan to meet a set of transportation requests by using a given fleet of vehicles at minimum cost.

In this paper, we aim at solving the DARP arising in the daily operation of the transportation sector of the municipal health care system in Ouro Preto, Brazil. Every day, the transportation department transfers patients from their pick-up location, which is either the patient's home or a collective point, to public hospitals located in the capital Belo Horizonte. Whether a patient is collected from their home or a public location (collective point) depends on the patient's condition. Patient transportation requests also differ regards to patient's vehicle requirement. That is, a transportation request can include either a single patient or a patient and a companion person and based on the patient's need (e.g. in a wheelchair) a specific v ehicle $\mathrm{m}$ ight $\mathrm{n}$ eed to b e allocated to the patient. To provide the transportation service, the sector leases a fleet of heterogeneous $\mathrm{v}$ e hicles. The vehicles have different (leasing) costs and load capacities. The main difference between the problem faced by the Ouro Preto's health care system and DARPs from the literature is that the proposed problem must have no transportation request rejected. That is, all patients must be served.

To solve this problem, we introduce a variable neighbourhood search (VNS)-based metaheuristic. The VNS method counts with a greedy method to find a in itial solution and a randomised va riable neighbourhood descent (RVND) method as the local search procedure. Solutions are evaluated using a cost function which includes routing cost and penalty cost for violating time window constraints. The VNS shaking procedure includes six inter-route operators, whereas the RVND method includes three. We calibrate the parameters of the VNS, namely neighbourhood combination, penalty for time window constraint violation, and stopping criteria, using a well-known calibration tool, the iRace package. We validate the performance of the proposed VNS on benchmark instances from Parragh (2011). We also create different test problems based on data provided by the public health care system and test the proposed approach on them. The computational experiments on Parragh (2011)'s instances show that our VNS is able to find optimal solutions in short computational times. When VNS was not able to find the optimal solution, the gap was less than $2.95 \%$. The results on the designed test problems indicate that by using the proposed approach, the transportation sector can lease fewer vehicles than it has been currently renting to attend daily patient transportation requests. We can conclude that the VNS metaheuristic is an efficient $t$ ool $\mathrm{f}$ or the $\mathrm{m}$ unicipal h ealth $\mathrm{c}$ are $\mathrm{t}$ ransportation s ector to i mprove routing and vehicle costs.
\end{abstract}

Keywords: Dial-a-ride problem, vehicle routing problem, patient transportation, variable neighbourhood search, randomised variable neighbourhood descent, optimisation 
M. Bernardo et al., The dial-a-ride problem in the case of a patient transportation...

\section{INTRODUCTION}

This paper was motivated by the problem faced by a municipal health care system in Brazil in its daily operation. The municipal health care system in Ouro Preto, like many other municipal health care systems in Brazil, is responsible for transporting patients to hospitals located in large cities. In Brazil, not only medical services but also the transportation of patients to public hospitals are provided by the municipality. Patients call the health care system to book a medical service and their transportation to the hospital where the service will be provided. Such transports start at the patient's home or a collective location and end at a specific hospital. For each transportation request, a time window for arrival at the hospital is specified based on the time of the medical appointment. After that, the health care system determines a time window for departure from the pick-up location. To provide the transport service, the health care system leases a fixed heterogeneous vehicle fleet. These vehicles are all located at the same depot and differ based on their load capacity and type of patient they can accommodate (e.g. stretcher). No patient transportation request can be rejected.

Our goal is to model the problem as a dial-a-ride problem (DARP), and then design solutions which improve routing and vehicle cost. The problem faced by the health care system can be classified as a variant of the Vehicle Routing Problem (VRP). The main difference between the DARP and other VRP variants is that the former considers the convenience and comfort of customers (Souza et al. 2021). In the DARP, multiple users make transportation requests from their specific origins to destinations. The transportation sector receives the requests, and then needs to manage its fleet to attend them (Ho et al. 2018). To solve the problem, we introduce a variable neighbourhood search (VNS)-based algorithm. This metaheuristic includes a local search procedure based on a randomised variable neighbourhood descent (RVND) method. The main difference between the proposed problem and DARPs from the literature is that in the former all patient transportation requests must be attended. We validate the performance of the proposed metaheuristic by comparing VNS results with those of Parragh (2011). We design a set of test problems based on data provided by the health care system, and then solve them with VNS.

The remainder of the paper is organised as follows. In the next section, we formally describe the patient transportation system and then formulate the mathematical model. The proposed solution approach is introduced in Section 3. The computational results on instances from the literature and on the real-life case study are displayed in Section 4, and the work is concluded in Section 5.

\section{THE PATIENT TRANSPORTATION SYSTEM}

In this section, firstly we provide a detailed description of the problem faced by Ouro Preto's health care system. After that, we present the notations and the mathematical formulation of the DARP.

\subsection{PROBLEM DESCRIPTION}

The problem faced by the public health care system is explained as follows. At the beginning of a work day the transportation department schedules transportation requests and calculates a route plan to be executed during that day. A transportation request includes either a single passenger (patient) or two passengers (patient plus companion). A companion person is required when the patient is unable to travel alone. A transportation request pick-up location is either the patient's home or collective points. Patients that are not able to move have to be collected at their homes, otherwise they are picked up at collective points. The collective points are distributed around the city and are located at specific points in the city, such as, big squares, old churches, and train stations. The collective point in which the patient has to be collected from is chosen based on the shortest distance between the patient's home and the collective point. Passengers must be collected at specific times such that they can arrive at their drop-off points on time for their medical treatments/services. Hospital arrival delays, however, can be be accepted. To attend patient transportation requests, the transportation department leases a fixed heterogeneous fleet of vehicles.

\subsection{MATHEMATICAL FORMULATION}

Let $G=(V, A)$ be a complete and undirected graph with node set $V=(P \cup D)$, which denote patient locations ( $v_{0}$ and $v_{2 n+1}$ represent the depot), and arc set $A(|A|=a)$. The set $V$ is subdivided into two sets, where $P=\{1, \ldots, n\}$ and $D=\{n+1, \ldots, 2 n\}$ are the pick-up and drop-off location sets, respectively. Then, $|V|=2 n$. Transportation cost $c_{i j}$ and travel time $\delta_{i j}$ are fixed for all $(i, j) \in A$. The heterogeneous vehicle fleet is represented by $K$. Each vehicle type $k \in K$ has a capacity $w^{k}$ and a cost $f^{k}$. Each patient $i \in P$ has a pick-up $i$ and drop-off $n+i$ locations, a load $q_{i}$, a non-negative service time $\tau_{i}$, and a time window 
$\left[e_{i}, l_{i}\right]$, where $e_{i}$ and $l_{i}$ are integers non-negative. A solution $y$, called route plan, is a set of routes. Each route is done by one vehicle which leaves the depot, serves a subset of users whose total load and total travel time do not exceed $w_{k}$ and $T_{k}$, respectively, and returns to the depot in the interval $\left[e_{0}, l_{0}\right]$. A customer must first be picked up and then delivered by the same vehicle. For every customer, the service should start between $\left[e_{i}, l_{i}\right]$. That is, let $A r_{i}^{k}$ denote the arrival time of vehicle $k$ at the vertex $i$, the beginning of the service $\left(B_{i}^{k}\right)$ cannot start before the beginning of the time window $\left(B_{i}^{k} \geq \max \left\{A r_{i}^{k}, e_{i}\right\}\right)$. The departure time $D_{i}^{k}=B_{i}^{k}+\tau_{i}$ is the time vehicle $k$ departures from customer $i$. Vehicle $k$ waiting time is defined by $W_{i}^{k}=B_{i}^{k}-A r_{i}^{k}$. The total duration of the route is calculated as $T_{k}=B_{n+1}^{k}-B_{0}^{k}$, where $B_{n+1}^{k}$ and $B_{0}^{k}$ represent when vehicle $k$ finishes and starts its ride at the depot, respectively. $x_{i j}^{k}$ is a binary variable that takes value 1 if edge $(i, j)$ is used by vehicle $k$ or zero otherwise. $w_{i}^{k}$ is an integer variable that takes the number of people in vehicle $k$ after visiting node $i$ or zero if the vehicle does not serve customer $i . L_{i}^{k}$ is a variable that takes the user ride time for patient $i$ in vehicle type $k$ or zero if the vehicle is not serving this patient. The formulation of the DARP is represented as follows. We follow the formulation from Cordeau (2006)'s.

$$
\begin{aligned}
& \min \sum_{k \in K} \sum_{i \in V} \sum_{j \in V} x_{i j}^{k} c_{i j}^{k}+\sum_{k \in K} \sum_{j \in V} f^{k} x_{0 j}^{k} \\
& \text { s.t. } \sum_{k \in K} \sum_{j \in V} x_{i j}^{k}=1 \text {, } \\
& \sum_{j \in V} x_{0 j}^{k}=1, \\
& \sum_{i \in V} x_{i 2 n+1}^{k}=1 \text {, } \\
& \sum_{j \in V} x_{i j}^{k}-\sum_{j \in V} x_{j n+i}^{k}=0, \\
& \sum_{i \in V} x_{i l}^{k}-\sum_{j \in V} x_{l j}^{k}=0, \\
& B_{j}^{k} \geq B_{i}^{k}+\tau_{i}+\delta_{i j}-M 1\left(1-x_{i j}^{k}\right), \\
& w_{j}^{k} \geq w_{i}^{k}+q_{i}-M 2\left(1-x_{i j}^{k}\right), \\
& L_{i}^{k}=B_{n+i}^{k}-\left(B_{i}^{k}+\tau_{i}\right), \\
& B_{2 n+1}^{k}-B_{0}^{k} \leq T_{k}, \\
& e_{i} \leq B_{i}^{k} \leq l_{i} \text {, } \\
& t_{\text {in }+1} \leq L_{i}^{k} \leq L \\
& \max \left\{0, q_{i}\right\} \leq w_{i}^{k} \leq \min \left\{w_{k}, w_{k}+q_{i}\right\}, \\
& x_{i j}^{k} \in\{0,1\} \text {, } \\
& \forall i \in P \text {, } \\
& \forall k \in K, \\
& \forall k \in K, \\
& \forall i \in P, \forall k \in K, \\
& \forall l \in V, \forall k \in K, \\
& \forall i \in V, \forall j \in V, \forall k \in K, \\
& \forall i \in V, \forall j \in V, \forall k \in K, \\
& \forall i \in V, k \in K, \\
& \forall k \in K, \\
& \forall i \in V, k \in K \text {, } \\
& \forall i \in P, k \in K \text {, } \\
& \forall i \in V, k \in K \text {, } \\
& \forall i \in V, j \in V, k \in K \text {. }
\end{aligned}
$$

The only difference between Cordeau (2006)'s model and the one above is that the objective function (1) minimises not only routing cost but also vehicle cost. Constraints (2), (3), and (4) ensure that all customers are served only once and all routes start and end at the depot. Constraint (5) guarantees a customer is attended by the same vehicle. The collection and drop-off flow of the vehicle are represented by Constraints (6). Constraints (7) and (8) state that the consistency of time variables and the vehicle capacity are respected, respectively, where $M 1 \geq \max \left\{0, l_{i}+\tau_{i}+\delta_{i j}-e_{j}\right\}$ and $M 2 \geq \min \left\{w_{k}, w_{k}+q_{i}\right\}$. Equalities (9) define the ride time of each user. Constraints (10) ensure that the total duration of route $k$ does not exceed a preset bound $T_{k}$. The starting time of the service at each node is guaranteed by (11). Constraints (12) guarantee the ride time of any user does not exceed $\mathrm{L}$. The ride time associated with request $i$ is computed as $L_{i}=B_{i+n}-D_{i}$. The vehicles capacity is represented by Constraints (13). Finally, constraints (14) set the domains of the binary decision variables.

\section{THE VARIABLE NEIGHBOURHOOD SEARCH ALGORITHM}

We developed a VNS-based algorithm to solve the problem. The pseudo-code of the greedy algorithm is shown in Algorithm 1. The algorithm starts with an initial solution $s$. To generate an initial solution, VNS employs a greedy method. The greedy method repeatedly allocates transportation requests to vehicle routes based on two variables: start of the time window and vehicle route duration. More precisely, the method inserts transportation request $i$ in the vehicle route whose duration $\left(T^{k}\right)$ is the closest to request's time window 
start $\left(e_{i}\right)$. After that, VNS generates a neighbour with the shaking procedure (line 4). We adopt six interroute shaking operators: relocation(1), relocation(2), $\operatorname{swap}(1), \operatorname{swap}(2), \operatorname{swap}(2,1)$ and cross. These operators are illustrated in Figure 1. The relocation(1) operator removes a customer from one route and inserts it into another route. The relocation(2) operator deletes two customers from one route and inserts them into another route. The swap(1) exchanges one customer from two selected routes, whereas swap(2) swaps two customers from two routes. The swap $(2,1)$ operator swaps two adjacent customers from one route and with one customer from another route. Finally, the cross operator exchanges two parts of two different routes while preserving the sequence of the requests in the exchanged route parts.
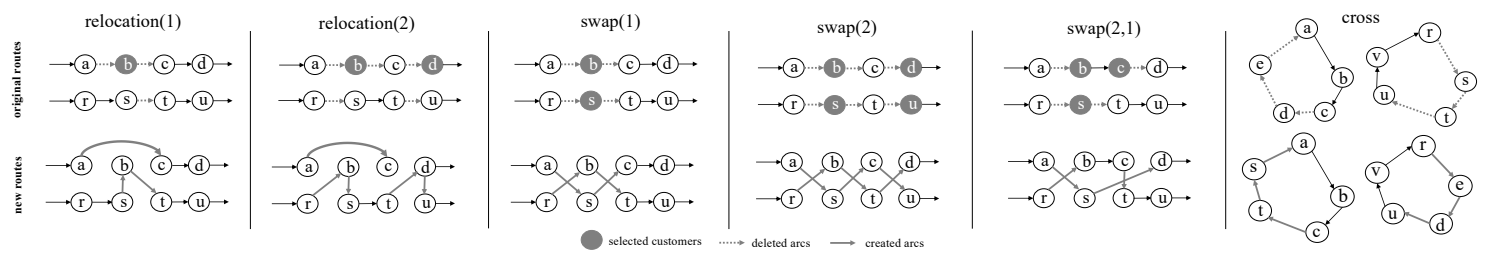

Figure 1. Shaking operators adopted in the VNS-based algorithm

A shaking operator is randomly selected for the shaking procedure, and the move is applied regardless of whether it incurs in an improvement in the solution. The neighbour is then improved by a local search procedure. We adopt a RVND method as the local search. The RVND method uses three inter-route operators. The operators selected in this method are presented in section 4.1. In RVND, the first neighbourhood is selected from the set $V$ of ordered neighbourhoods (line 6). Then, the current solution $s$ is submitted to a local search in $V_{i}$ with the first improvement strategy (line 7). If the resulting solution $s^{\prime}$ from the local search is better than the current solution $s$, i.e. the solution found by RVND is better than the incumbent solution (line 8), then $s$ is updated. After that, a new order of neighbourhoods is randomly generated (10) and a new iteration is performed with the first neighbourhood in the set. That is, the search will start from the first neighbourhood in the set. If the solution found by RVND is worse than the incumbent solution, $V_{i}$ is removed from the set (line 12). Then, the next neighbourhood in the set is selected and a new iteration is done with the unchanged current solution. The stopping criterion is defined by the number of iterations without improvement in the current solution (iter Max $=1000$ ).

Solutions are evaluated using a cost function $f(s)=c(s)+\alpha t w(s)+\beta t(s)+\pi d(s)+\gamma q(s)$, where $c(s)$ is the total routing cost and $t w(s), t(s), d(s)$ and $q(s)$ denote the total violation of time window, ride time, duration, and load constraints, respectively (Cordeau $\&$ Laporte 2003). The total violation of load and duration constraints is computed on a route basis with respect to $Q_{k}$ and $T_{k}$, whereas the total violation of time window constraints is equal to $\sum_{i=0}^{2 n}\left(B_{i}-l_{i}\right)^{+}$, where $x^{+}=\max \{0, x\}$. Similarly, the total violation of ride time constraints is equal to $\sum_{i=1}^{n}\left(L_{i}-L\right)^{+}$. The penalisation variables for time window $(\alpha)$, ride time $(\beta)$, load $(\gamma)$ and duration $(\pi)$ violations were set to $\alpha=\beta=\pi=\gamma=1$.

\section{COMPUTATIONAL EXPERIMENTS}

In this section, we present the results obtained with the proposed solution approach on benchmark instances and on instances designed based on real data provided by Ouro Preto's health care system. All experiments were implemented in $\mathrm{C}++$ and performed on an Intel Core i7-7700U CPU @ 3.40 GHz x 4 with 16GB RAM. The code and the solutions are available to interested readers upon request.

\subsection{PARAMETERS CALIBRATION}

The performance of the VNS algorithm is highly affected by the values of its parameters. It is then important to calibrate the best values for the algorithm parameters so that the algorithm can reach its best performance. We determine suitable values for the VNS parameters by using a well-known generic calibration tool, the $i$ Race package proposed by López-Ibáñez et al. (2016). Readers are referred to López-Ibáñez et al. (2016) for more details about the iRace package. The parameters of the VNS that were tuned together with the set of possible values are presented in Table 1. The VNS algorithm adopts six inter-route shaking operators, whereas the RVND (local search procedure) uses only three inter-route operators (three neighbourhood structures). We then consider the six inter-route shaking operators as the pool of options for calibrating the RVND neighbourhood structures. The options for the three inter-route RVND operators are represented as follows: relocation(1) - 1, swap(1) - 2, cross - 3, swap(2) - 4, relocation(2) - 5 and swap(2,1) - 6. PI represents the coefficient of the penalty cost functions in evaluation function $f(s)$. 


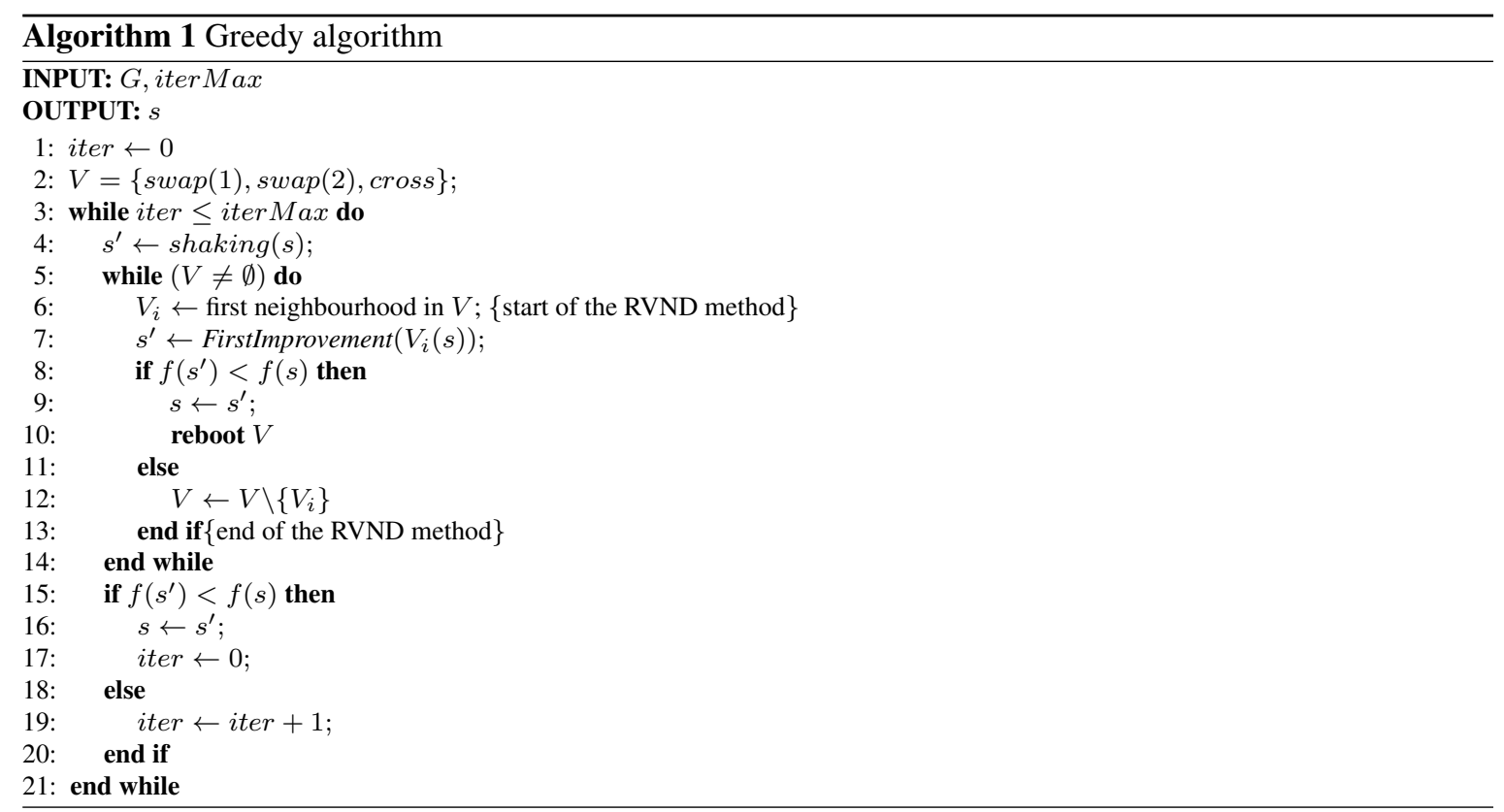

Table 1. The parameters calibrated by iRace in the VNS algorithm

\begin{tabular}{cccc}
\hline Parameter & Description & Set of values & Final value \\
\hline NC & Neighbourhood combination & $\{123,124,125,126,134,135,136,145,146,156,234$, & 234 \\
PI & Penalty cost functions' coefficients & $235,236,245,246,256,345,346,356,456\}$ & 10 \\
iterMax & VNS stopping criteria & $\{1,3,5,7,10\}$ & 100 \\
\hline
\end{tabular}

\subsection{INSTANCES DESCRIPTION}

To validate the performance of the VNS-based algorithm, we solve the benchmark instances from Parragh (2011) and compare the VNS results with those of Parragh (2011) and Braekers et al. (2014). Parragh (2011)'s instances were modified from Cordeau (2006)'s instances. Parragh (2011) designed instances with both homogeneous and heterogeneous vehicle fleets. The benchmark dataset includes three groups of instances (E, I and $\mathrm{U}$ ). The name of the test problem is defined as $a k-n$, where $k$ represents the number of vehicle available in the instance and $n$ describes the instance's number of transportation requests. Each group has 12 instances with 2-4 vehicles and 16-48 transportation requests. Transportation requests vary based on types of passengers and each request consists of at most one patient. Passengers are classified as seated, on stretcher or in wheelchair. In each instance group a different fleet configuration is used. In all instances, the time window length is $l_{i}-e_{i}=15$ minutes, the maximum user ride time $L=30$ minutes, and service time $\tau_{i}=3$ minutes for all passengers. All designed instances are available for download in Souza (2019).

\subsection{RESULTS ON BENCHMARK INSTANCES}

Table 2 displays the computational results for the benchmark instances proposed by Parragh (2011). The column Opt shows the optimal objective function value for each instance obtained from the work of Braekers et al. (2014). The columns under Parragh (2011) present the results obtained by Parragh (2011), whereas the columns under VNS display the results obtained with the proposed solution approach. Best sol is the objective function value of the best solution, \%Gap is the gap from the optimal objective function value, Avg sol is the average objective function value in 10 runs, Avg \%gap is the gap for the Avg sol from the optimal objective function value, and Time is the computational time, in seconds, for finding the best solution (in seconds). We can see from the results that the Parragh (2011)'s solution approach outperformed VNS. The former was able to find 25 optimal solutions, whereas the latter found 10 optimal solutions. Nevertheless, VNS was faster than Parragh (2011)'s approach in 29 instances. The worst VNS gap was only $2.95 \%$. 
M. Bernardo et al., The dial-a-ride problem in the case of a patient transportation...

Table 2. Computational results on benchmark dataset

\begin{tabular}{|c|c|c|c|c|c|c|c|c|c|c|}
\hline \multirow[t]{2}{*}{ Group } & \multirow[t]{2}{*}{ Instance } & \multirow[t]{2}{*}{ Opt } & \multicolumn{3}{|c|}{ Parragh } & \multicolumn{5}{|c|}{ VNS } \\
\hline & & & Best sol & Gap & Time & Best sol & $\%$ Gap & Avg sol & Avg \% gap & Time \\
\hline \multirow{12}{*}{$\mathrm{U}$} & a2-16 & 294.25 & 294.25 & 0.00 & 68.20 & 294.25 & 0.00 & 294.25 & 0.00 & 3.83 \\
\hline & a2-20 & 344.83 & 344.83 & 0.00 & 133.80 & 344.83 & 0.00 & 344.83 & 0.00 & 15.69 \\
\hline & a2-24 & 431.12 & 431.12 & 0.00 & 187.80 & 434.53 & 0.78 & 436.48 & 1.23 & 42.26 \\
\hline & a3-18 & 300.48 & 300.48 & 0.00 & 45.40 & 300.48 & 0.00 & 302.53 & 0.68 & 2.60 \\
\hline & a3-24 & 344.83 & 344.83 & 0.00 & 86.80 & 344.91 & 0.02 & 347.50 & 0.77 & 14.58 \\
\hline & a3-30 & 494.85 & 494.85 & 0.00 & 105.60 & 500.51 & 1.13 & 502.52 & 1.53 & 74.02 \\
\hline & a3-36 & 583.19 & 583.30 & 0.02 & 162.60 & 599.43 & 2.71 & 607.24 & 3.96 & 247.54 \\
\hline & a4-16 & 282.68 & 282.68 & 0.00 & 26.00 & 283.10 & 0.15 & 283.10 & 0.15 & 1.04 \\
\hline & a4-24 & 375.02 & 375.02 & 0.00 & 50.80 & 379.36 & 1.14 & 380.81 & 1.52 & 6.36 \\
\hline & a4-32 & 485.50 & 486.88 & 0.28 & 86.00 & 487.31 & 0.37 & 491.70 & 1.26 & 54.24 \\
\hline & a4-40 & 557.69 & 561.80 & 0.74 & 130.60 & 557.69 & 0.00 & 569.36 & 2.05 & 243.66 \\
\hline & a4-48 & 668.82 & 673.64 & 0.72 & 253.80 & 678.98 & 1.50 & 683.13 & 2.09 & 580.92 \\
\hline \multirow{12}{*}{ E } & a2-16 & 331.16 & 331.16 & 0.00 & 65.60 & 331.16 & 0.00 & 331.16 & 0.00 & 4.89 \\
\hline & a2-20 & 347.03 & 347.03 & 0.00 & 120.00 & 347.03 & 0.00 & 347.03 & 0.00 & 16.32 \\
\hline & a2-24 & 450.25 & 450.25 & 0.00 & 160.40 & 450.38 & 0.02 & 452.92 & 0.65 & 76.08 \\
\hline & a3-18 & 300.63 & 300.63 & 0.00 & 47.60 & 300.63 & 0.00 & 302.01 & 0.46 & 2.05 \\
\hline & a3-24 & 344.91 & 344.91 & 0.00 & 76.20 & 344.91 & 0.00 & 347.63 & 0.86 & 17.73 \\
\hline & a3-30 & 500.58 & 500.58 & 0.00 & 107.60 & 505.64 & 1.00 & 507.72 & 1.54 & 108.75 \\
\hline & a3-36 & 583.19 & 583.19 & 0.00 & 161.60 & 599.43 & 2.71 & 610.46 & 4.46 & 316.76 \\
\hline & a4-16 & 285.99 & 285.99 & 0.00 & 25.00 & 291.55 & 1.91 & 291.76 & 1.98 & 1.25 \\
\hline & a4-24 & 383.84 & 383.84 & 0.00 & 52.60 & 386.06 & 0.57 & 289.09 & 1.35 & 8.60 \\
\hline & a4-32 & 500.24 & 500.52 & 0.45 & 83.00 & 501.85 & 0.32 & 507.11 & 1.35 & 49.16 \\
\hline & a4-40 & 580.42 & 585.64 & 0.90 & 121.00 & 589.29 & 1.51 & 597.89 & 2.92 & 204.60 \\
\hline & a4-48 & 670.52 & 675.37 & 0.72 & 252.20 & 676.28 & 0.85 & 688.57 & 2.62 & 786.57 \\
\hline \multirow{13}{*}{ I } & a2-16 & 294.25 & 294.25 & 0.00 & 68.40 & 294.25 & 0.00 & 294.25 & 0.00 & 5.33 \\
\hline & a2-20 & 355.74 & 355.74 & 0.00 & 141.80 & 360.23 & 1.25 & 362.69 & 1.92 & 14.16 \\
\hline & a2-24 & 431.12 & 431.12 & 0.00 & 211.00 & 424.53 & 0.78 & 441.32 & 2.31 & 59.08 \\
\hline & a3-18 & 302.17 & 302.17 & 0.00 & 47.20 & 302.17 & 0.00 & 303.27 & 0.36 & 3.53 \\
\hline & a3-24 & 344.83 & 344.83 & 0.00 & 83.60 & 345.31 & 0.14 & 350.79 & 1.70 & 19.23 \\
\hline & a3-30 & 494.85 & 494.85 & 0.00 & 106.80 & 502.77 & 1.57 & 511.75 & 3.30 & 93.29 \\
\hline & a3-36 & 618.15 & 618.58 & 0.07 & 170.60 & 636.97 & 2.95 & 641.98 & 3.71 & 296.38 \\
\hline & a4-16 & 299.05 & 299.05 & 0.00 & 27.00 & 302.87 & 1.26 & 307.23 & 2.66 & 1.09 \\
\hline & a4-24 & 375.02 & 375.07 & 0.01 & 51.60 & 379.36 & 1.14 & 381.91 & 1.80 & 9.83 \\
\hline & a4-32 & 486.93 & 486.93 & 0.00 & 88.00 & 488.74 & 0.37 & 496.25 & 1.88 & 59.96 \\
\hline & a4-40 & 557.69 & 561.35 & 0.66 & 132.20 & 566.11 & 1.49 & 573.02 & 2.68 & 279.11 \\
\hline & a4-48 & 670.72 & 680.43 & 1.45 & 262.40 & 684.37 & 1.99 & 695.95 & 3.63 & 749.48 \\
\hline & & & Average & 0.16 & & & 0.82 & 1.64 & & \\
\hline
\end{tabular}

\subsection{RESULTS ON REAL-CASE INSTANCES}

In this work, we study the patient transportation problem faced by transportation sector of the Ouro Preto's health care system. We therefore created a set of four test problems based on real data provided by the sector, to evaluate the proposed solution approach. The provided data show a schedule of some requests served by the transportation sector. These requests are related to workdays occurred between March and October 2019. Each transportation request includes number of customers, pick-up and drop-off locations, and time windows. Two instances, named a9-21 and a9-24, were designed based on information from two working days. Instance a9-34 was created by joining four working days. The largest instance (a9-48) was designed to simulate a really busy working day. In this instance half of the pick-up locations were patients' homes and the other half was collective points. For generating the patient's home locations, we randomly selected points in the municipal area and for generating each one of the locations of the collective points we randomly selected one of the six collective points provided by the transportation sector. To attend patient transportation requests, the transportation sector leases a fleet composed of small cars, ambulances, and a van. Each vehicle has a different capacity and a cost (leasing) associated with it. Table 3 presents the characteristics of each vehicle.

Table 3. Fleet of vehicles features

\begin{tabular}{ccccccc}
\hline & & & & \multicolumn{3}{c}{ Number of passengers } \\
\cline { 5 - 7 } Vehicle & Representation & Capacity & Cost & Non-stretcher & Stretcher & Companion \\
\hline Small car 1 & S1 & 4 & 100 & 3 & 0 & 2 \\
Small car 2 & S2 & 5 & 120 & 2 & 0 & 2 \\
Ambulance 1 & A1 & 1 & 100 & 0 & 1 & 2 \\
Ambulance 2 & A2 & 4 & 150 & 0 & 2 & 2 \\
Van & V & 16 & 200 & 8 & 0 & 8 \\
\hline
\end{tabular}

The computational results are presented in Table 4. The columns under Drop-off show the number of patient's home and collective points in each instance. The columns Sol, RC, VC, Used vehicles, Time display the objective function value, routing cost, vehicle cost, vehicles adopted in the best solution found in 10 runs, and computational time for finding the best solution (in seconds), respectively. The computational experiments 
show that the objective function value is split almost in half between routing and vehicle costs. We can also notice that none of the best solutions required using all of the vehicles in the fleet to attend the transportation requests. Finally, the proposed solution approach is able to find good quality solutions for all of the designed test problems, and in a short computational time.

Table 4. Computational results on the designed test problems

\begin{tabular}{|c|c|c|c|c|c|c|c|c|c|c|}
\hline \multirow[t]{2}{*}{ Test problem } & \multicolumn{2}{|c|}{ Drop-off } & \multicolumn{2}{|c|}{ Sol } & \multicolumn{2}{|c|}{$\mathrm{RC}$} & \multicolumn{2}{|c|}{$\mathrm{VC}$} & \multirow[t]{2}{*}{ Used vehicles } & \multirow[t]{2}{*}{ Time } \\
\hline & Pat.'s home & Col. point & Best & Avg & Best & Avg & Best & Avg & & \\
\hline a9-21 & 18 & 3 & 941.82 & 1041.78 & 501.82 & 526.78 & 440 & 515 & $1 \mathrm{xS} 1,2 \mathrm{xS} 2,1 \mathrm{xA} 1$ & 19.40 \\
\hline a9-24 & 19 & 5 & 980.90 & 1108.42 & 540.90 & 513.42 & 440 & 595 & 1xS1, 2xS2, 1xA1 & 104.17 \\
\hline a9-34 & 20 & 14 & 1136.68 & 1325.03 & 566.68 & 635.03 & 570 & 690 & 1xS1, 1xS2, 1xA2, 1xV & 510.36 \\
\hline a9-48 & 24 & 24 & 1764.18 & 1858.78 & 974.18 & 970.78 & 790 & 888 & $3 \mathrm{xS} 1,4 \mathrm{xS} 1,1 \mathrm{xS} 2,1 \mathrm{xA} 2$ & 3682.11 \\
\hline
\end{tabular}

\section{CONCLUSIONS}

In this study, we address the patient transportation problem faced by the transportation sector of the Ouro Preto's health care system. The problem can be modelled as a DARP where the fleet of vehicles is heterogeneous. Patient transportation requests differ regards to number of passengers (single patient or patient with companion), vehicle requirement (seated, on stretcher or in wheelchair), pick-up and drop-off locations, and type of drop-off location (patient's home or collective point). We introduce a VNS-based metaheuristic to solve the proposed problem. The algorithm employs a greedy method to generate an initial solution and a RVND method as the local search procedure. The performance of the VNS approach is validated by comparing its results with those of Parragh (2011) on benchmark instances. The Parragh (2011)'s algorithm outperformed the VNS metaheuristic regards to solution quality, but the latter was far faster than the former. However, it is important to highlight that the computers used in these two studies are quite different, and we could therefore obtain a different result when converting computer specifications one to another. For the Parragh (2011)'s instances which the VNS algorithm was not able to find the optimal solution, the gap was no greater than $2.95 \%$. Therefore, we can conclude that the proposed solution approach is efficient to solve real-case problems, such as the one faced by the Ouro Preto's health care transportation department, as the sector does not attend more than 25 transportation requests in a working day. The results on instances designed from real data show that not all vehicles are used in the solutions found by VNS. This means that the transportation sector does not need to lease the same fleet it has been leasing every working day. The proposed VNS can then be adopted by the transportation sector as a fleet management tool to improve routing and vehicle costs.

\section{ACKNOWLEDGEMENT}

We also would like to acknowledge both the Federal University of Ouro Preto and the transportation sector of the local health system of Ouro Preto.

\section{REFERENCES}

Braekers, K., Caris, A. \& Janssens, G. K. (2014), 'Exact and meta-heuristic approach for a general heterogeneous dial-a-ride problem with multiple depots', Transportation Research Part B: Methodological 67, 166-186.

Cordeau, J.-F. (2006), 'A Branch-and-Cut Algorithm for the Dial-a-Ride Problem', Operations Research 54(3), 573-586.

Cordeau, J. F. \& Laporte, G. (2003), 'The Dial-a-Ride Problem (DARP): Variants, modeling issues and algorithms', Quarterly Journal of the Belgian 1, 89-101.

Ho, S. C., Szeto, W. Y., Kuo, Y. H., Leung, J. M. Y., Petering, M. \& Tou, T. W. H. (2018), 'A survey of dial-a-ride problems: Literature review and recent developments', Transportation Research Part B: Methodological 111, 395-421.

López-Ibáñez, M., Dubois-Lacoste, J., Pérez, L. C., Birattari, M. \& Stützle, T. (2016), 'The Irace package: Iterated racing for automatic algorithm configuration', Operations Research Perspectives 3, 43-58.

Parragh, S. N. (2011), 'Introducing heterogeneous users and vehicles into models and algorithms for the diala-ride problem', Transportation Research Part C: Emerging Technologies 19(5), 912-930.

Souza, A. L. S. (2019), 'Dial-a-Ride Problem (DARP) dataset from Ouro Preto-MG, Brazil', Mendeley Data .

Souza, A. L. S., Bernardo, M., Penna, P. H. V., Pannek, J. \& Souza, M. J. F. (2021), 'Bi-objective optimization model for the heterogeneous dynamic dial-a-ride problem with no rejects', Optimization Letters pp. 1-20. 